\title{
TRATAMENTO CIRÚRGICO DO SCHWANNOMA TRAQUEAL
}

\author{
SURGICAL TREATMENT OF TRACHEAL SCHWANNOMA
}

\section{INTRODUÇÃO}

Os tumores primários traqueais são neoplasias raras, encontradas em cerca de $0,1 \%$ das doenças malignas e são 180 vezes menos freqüentes que os de pulmão ${ }^{1}$. São representadas, na maior parte das vezes, por dois principais tipos histológicos: carcinoma de células escamosas e carcinoma adenóide cístico ${ }^{2}$. As manifestações clínicas são inespecíficas, prejudicando muito o diagnóstico precoce e as modalidades de tratamento ainda não são consensuais $^{3-5}$. Os tumores benignos da traquéia são muito menos freqüentes e compreendem um grupo heterogêneo. $\mathrm{O}$ schwannoma é uma neoplasia benigna extremamente rara, e até 1983 poucos casos estavam relatados na literatura ${ }^{4}$. Devido à raridade do tumor e ao seu crescimento lento, o retardo médio no diagnóstico é de 10 a 15 meses do início dos sintomas. Neste momento não é incomum que $50 \%$ a $75 \%$ da luz da traquéia esteja ocluída pelo tumor. $\mathrm{O}$ diagnóstico é baseado em sintomas inespecíficos como tosse persistente, broncoespasmo e estridor, que pode ser confirmado por laringotraqueobroncoscopia e tomografia computadorizada de tórax. O tratamento consiste em ressecção cirúrgica ou endoscópica. Pretendemos neste estudo apresentar um caso de schwannoma traqueal, tratado por ressecção cirúrgica.

\section{RELATO DO CASO}

Paciente de sexo feminino, 16 anos, branca, foi admitida na Disciplina de Clínica Cirúrgica III da Universidade Federal do Maranhão com quadro de dispnéia, broncoespasmo, tosse e pneumonia de repetição há dois anos. Negava história de tabagismo. Ao exame físico se apresentava com estado geral e de nutrição regulares e à ausculta do aparelho respiratório foram observados sibilos e

\author{
Orlando Jorge Martins Torres, TCBC-MA ${ }^{1}$ \\ Nelson Lúcio Parada Martins, TCBC-MA ${ }^{2}$ \\ Ricardo Maurício Batista Feitosa Cuba ${ }^{3}$
}

sinais de broncoespasmo difuso bilateralmente. Realizou radiografia de tórax em incidência póstero-anterior e perfil que estava sem alterações. Nos exames laboratoriais a paciente apresentava leucocitose de $11.700 / \mathrm{mm}^{3}$ com $69 \%$ de segmentados, sem outras alterações. Em seguida, a paciente foi submetida a uma broncoscopia que evidenciou lesão polipóide na parede lateral da traquéia, ocupando aproximadamente 4/5 da luz do órgão (Figura 1). A tomografia computadorizada (TC) da região cervical e tórax mostrou lesão sólida na luz traqueal, porém sem comprometimento mediastinal ou envolvimento linfonodal (Figura 2).

Após preparo pré-operatório, a paciente foi submetida a tratamento cirúrgico consistindo em cervicotomia transversa anterior, com exposição e abertura horizontal

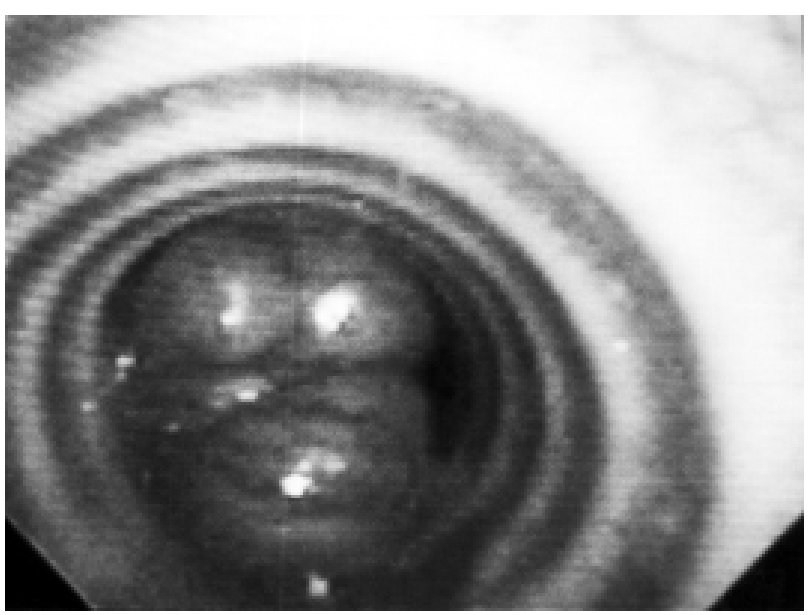

Figura 1 - Broncoscopia: lesão polipóide na parede lateral da traquéia.

1. Professor Livre-Docente e Coordenador da Disciplina de Clínica Cirúrgica III - UFMA

2. Professor do Departamento de Cirurgia e Cirurgião de Tórax - UFMA

3. Cirurgião Geral

Recebido em 15/05/2001

Aceito para publicação em 17/09/2002

Trabalho realizado na Disciplina de Clínica Cirúrgica III da Universidade Federal do Maranhão - UFMA 


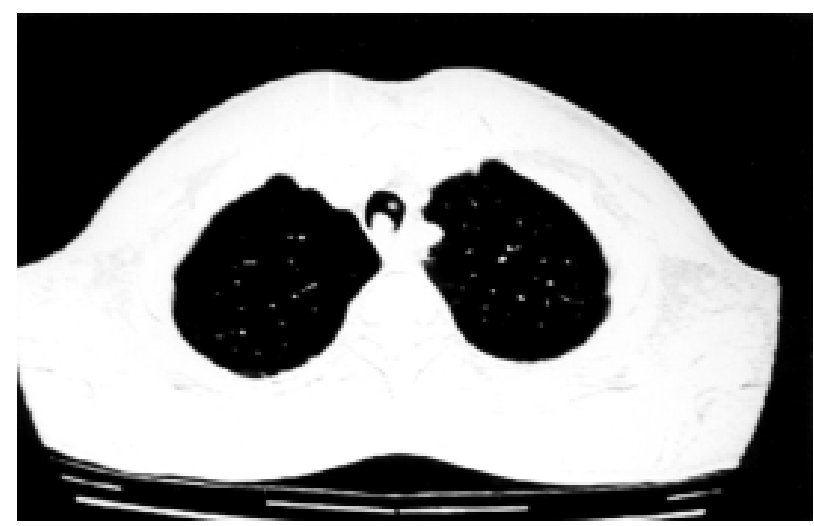

Figura 2 - Tomografia computadorizada da região cervical: lesão sólida na luz traqueal.

da traquéia ao nível do sexto anel traqueal, com ressecção deste e do sétimo anel juntamente com neoplasia, que apresentava macroscopicamente aspecto brancacento e friável durante a manipulação. A seguir foi realizada anastomose terminoterminal da traquéia e síntese anatômica dos planos musculares da região cervical. $\mathrm{O}$ maior fragmento da peça operatória mediu $2,0 \mathrm{~cm}$ x $0,8 \mathrm{~cm}$ e o estudo anatomopatológico mostrou proliferação de células fusiformes ou arredondadas com amplos citoplasmas. Estas células se dispunham em feixes contendo vasos congestos de permeio, sendo a lesão diagnosticada como schwannoma. A paciente evoluiu de forma satisfatória, permanecendo em unidade de terapia intensiva por 24 horas e, em seguida, encaminhada para a enfermaria, recebendo alta hospitalar no $7^{\circ}$ dia do período pós-operatório sem intercorrências. A paciente retornou para seguimento após um ano do procedimento cirúrgico e permanecia assintomática.

\section{DISCUSSÃO}

As neoplasias benignas da traquéia correspondem, no máximo, a 1,5\% do total de neoplasias que acometem este órgão. As neoplasias malignas representam a grande maioria dos tumores da traquéia, onde o carcinoma epidermóide e o carcinoma adenóide cístico representam, juntos, aproximadamente $65 \%$ do to$\mathrm{tal}^{1,2}$. As neoplasias benignas são incomuns em adultos e mais ainda em crianças e os sintomas mais freqüentes registrados na literatura são a tosse, dispnéia, hemoptise, estridor e broncoespasmo, sendo que aqueles mais específicos para doença traqueal são o estridor, a síndrome da veia cava superior e a disfagia ${ }^{2,3}$. Os pacientes freqüentemente procuram assistência médica devi- do a um quadro de "asma". Desta forma, quase sempre o diagnóstico é tardio, ocorrendo em média dez meses após o início dos sintomas e é, geralmente, concluído após exame endoscópico. O diagnóstico de obstrução de via aérea superior pode ser sugestivo e, neste caso, a avaliação espirométrica é útil, concluindo-se com o exame endoscópico. O diagnóstico diferencial de uma massa traqueal inclui neoplasias malignas (carcinoma de células escamosas, carcinoma adenóide cístico e carcinóide) e os tumores benignos como papiloma, hemangioma, hamartoma, schwannoma e neurofibroma ${ }^{1,3,4}$.A radiografia de tórax, tomografia computadorizada da região cervical e do tórax e cintilografia óssea são também elementos úteis no diagnóstico ${ }^{1,2}$.

O tratamento varia muito de acordo com o serviço, porém as principais modalidades de tratamento são a cirurgia, a radioterapia, a quimioterapia e as ressecções endoscópicas ${ }^{2-4}$. Nas doenças malignas da traquéia predomina a radioterapia, reservando-se a cirurgia para alguns poucos casos. No caso das doenças benignas, como no presente estudo, as ressecções endoscópicas e a cirurgia são as mais indicadas ${ }^{3,5}$. No caso específico do schwannoma, o tratamento de escolha é a ressecção cirúrgica com dois a três anéis traqueais e reconstrução da traquéia, e a ressecção endoscópica a laser também tem sido usada com sucesso neste tipo de lesão $0^{4,5}$.

Schwannoma traqueal foi primeiro descrito em 1951 por Straus e Guckien e foram registrados 20 casos até 1998, a maioria em adultos. Estes tumores são geralmente únicos, encapsulados, compostos por células schwann ligados ao nervo, porém sem conter neurônios. Normalmente os schwannomas não apresentam degeneração maligna. São mais freqüentemente localizados no terço distal da traquéia e se apresentam com sintomas de tosse e estridor. Muitos pacientes podem ser tratados por longo tempo como portadores de asma ${ }^{4}$. Nestas situações o diagnóstico diferencial deve ser realizado precocemente para não haver retardo no tratamento. O diagnóstico diferencial nestes pacientes também deve ser feito com corpo estranho na traquéia, fibrose cística, deficiências imunológicas, tranqueobroncomalácia e doença do refluxo gastroesofágico com aspiração ${ }^{3,4}$. Estes tumores têm sido normalmente tratados com ressecção endoscópica, resecção cirúrgica através de fissura traqueal ou laríngea, bem como ressecção traqueal ${ }^{2-4}$.

Uma vez que estes tumores podem recorrer, tem sido sugerido o seguimento destes através de provas de função pulmonar e exames de imagem. O prognóstico é influenciado muito pouco pelos sintomas e pela localização do tumor, porém a variedade histológica e a idade do paciente no momento do diagnóstico são fatores decisivos ${ }^{2,3}$. 


\section{ABSTRACT}

Schwannoma is a rare benign tumor of the proximal tracheobronchial tree. The aim of the present study is to report a case of tracheal schwannoma causing airway obstruction. A 16-year-old woman complained of cough, wheezing and dyspneia. Bronchoscopy and computerized tomography showed a polypoide intratracheal mass obstructing approximately $80 \%$ of the lumen. The treatment consisted of tracheal resection and primary anastomosis. Histological analysis revealed a tracheal schwannoma. The postoperative course was uneventful and the patient remains well twelve months after surgery.

Key Words: Tracheal neoplasm; Schwannoma; Tracheal surgery

\section{REFERÊNCIAS}

1. Grillo HC, Mathisen DJ. Primary tracheal tumors: treatment and results. Ann Thorac Surg 1990;49:69-77.

2. Dorfman J, Jamison BM, Morin JE. Primary tracheal Schwannoma. Ann Thorac Surg 2000;69:280-281.

3. Straus GD, Guckien JL. Schwannoma of the tracheobronchial tree. Ann Otol Rhinol Laryngol 1951;60:242246

4. Weiner DJ, Weatherly RA, Dipietro MA et al. Tracheal Schwannoma presenting as status asthmaticus in a sixteen-year-old boy. Pediatr Pneumol 1998;25:393397.
5. Rusch VW, Schmidt RA. Tracheal Schwannoma: management by endoscopic laser resection. Thorax 1994; 49:85-86.

Endereço para Correspondência:

Orlando Torres

Rua Ipanema, 1 - Ed. Luggano Bl 1/204 - São Francisco 65076-060 - São Luís-MA

E-mail: otorres@elo.com.br 